\title{
The Evolution of High Impedance Fault Modeling
}

\author{
Naser Zamanan \\ Dept. of Electrical Engineering \\ College of Technological Studies \\ Kuwait
}

\author{
Jan Sykulski \\ School of Electronics \& Computer Science \\ University of Southampton
}

U.K

\begin{abstract}
There is an increasing demand for more detailed and accurate modeling techniques for predicting transient response of power systems caused in particular by high impedance arcing faults (HIF). This is particularly so in relation to the design and development of improved equipment and new protection techniques. Accurate prediction of fault transients requires detailed and comprehensive representation of all components in a system, while the transient studies need to be conducted into the frequency range well above the normal power frequency. The HIF is a very complex phenomenon and exhibits highly nonlinear behavior. The most distinctive characteristics are nonlinearity and asymmetry. The nonlinearity arises from the fact that the voltage-current characteristic curve of the HIF is itself nonlinear. It is also observed that the fault current has different waveforms for positive and negative half cycles, which is called asymmetry. The nonlinearity and asymmetry exist in every cycle after the HIF. In order to obtain a good representation of a HIF, it is necessary to develop a model that gives the above mentioned characteristics, as well as the harmonic content of the HIF. This paper introduce a new HIF model, it also reviews and summarizes some of the methods for modeling high impedance faults and the developments which led to them.
\end{abstract}

Index Terms-asymmetry, Distribution Networks, Fault detection, HIF, HIF modeling, nonlinearity.

\section{INTRODUCTION}

A High Impedance Fault (HIF) on a distribution feeder is an abnormal circuit condition which results in energy being dissipated in a manner other than the serving of the intended load. Most faults can be detected and isolated in time, but high impedance faults and arcing faults are still difficult to detect in practice [2] because the currents the faults produce are small and they mimic normal loads. Distribution feeder conductors are prone to physical contact with neighboring objects such as overgrown vegetation, building walls, asphalt, a high impedance object or surface, which limits current values for faults on distribution systems and prevents conventional overcurrent protection from operating. In the case of an arcing HIF, when an energized conductor contacts the ground, the electric contact is not solid. Due to the existence of air between ground and conductor, the high potential difference in such a short distance excites the appearance of the arc. High impedance faults (HIF) have characteristics in their transient and steady state regimes that make them identifiable. They also lead to arcing and it is the result of air gaps due to the poor contact made with the ground or grounded objects; it occurs when a conductor breaks and falls on a non-conducting surface such as asphalt road, sand, cement, grass or perhaps a tree limb,

The financial support from the Public Authority of Applied Education and Training (PAAET) in Kuwait is highly appreciated. producing very little if any measurable current. The arcing that often results from HIFs can have deadly fire and electrocution consequences. A HIF may result in damage to the electrical system, loss of power to customers, public hazard, or possible unsafe conditions due to arcing and flashing and possible property fire.

An accurate modeling method for a HIF is essential for the development of reliable detecting algorithms. The HIF model's data should contain the complex characteristics of HIF such as nonlinearity, asymmetry, and the low frequency phenomena typical of an arcing fault. Some modeling methods of HIFs have been proposed [1-4]. Although all these methods represent well the nonlinear behavior of HIFs, difficulty arises when the other characteristics are considered.

According to the experimental work of the Korea Electric Power Corporation (KEPCO) [4], the HIF experimental data was collected on a $22.9 \mathrm{kV}$ distribution system. The total number of experiments was thirty two and the sampling frequency $10 \mathrm{kHz}$. Figure 1 exemplifies the currents for the $20^{\text {th }}$ and $40^{\text {th }}$ cycle after the HIF has occurred; both currents exhibit some asymmetry. Figure 2 shows the voltage-current curve and demonstrates the degree of nonlinearity. We can see that the "signature" of the current curve and the currentvoltage curve for HIF has a very unique shape. While designing a model for HIF, it must be taken in consideration that the developed model should give the above mentioned characteristics and harmonic content of HIF.

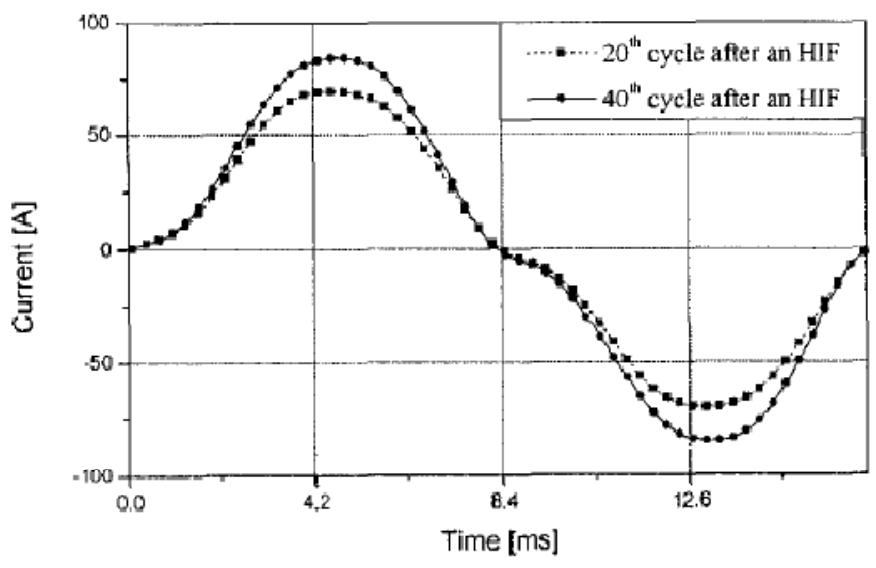

Figure 1. Currents for the $20^{\text {th }}$ and $40^{\text {th }}$ cycle after HIF (asymmetry) [4] 


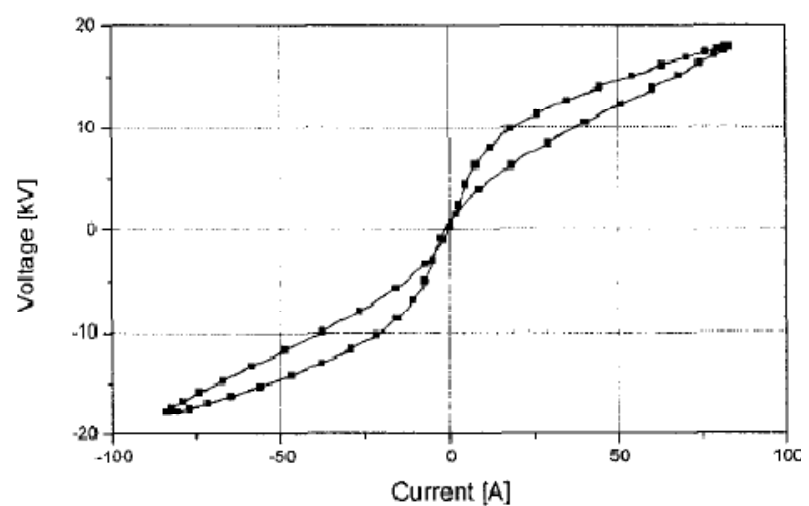

Figure 2. Voltage-current characteristic curve for one cycle in the steady state after HIF (nonlinearity) [4]

\section{REVIEW OF HIF MODELS}

Arcing associated with the HIFs results in energy dissipation in the form of heat that turns the moisture in the soil into steam and burns the grass into smoke. In the arcing phenomenon associated with downed power lines, due to the existence of air between ground and conductor, the high potential difference in such a short distance excites the appearance of the arc. Also, arcing often accompanies these faults, which poses a fire hazard. Therefore, from both public safety and operational reliability viewpoints, detection of HIFs is critically important. High impedance fault is a difficult case to model because most HIF phenomena involve arcing, which has not been perfectly modeled so far. Some previous researchers have reached a consensus that HIFs are nonlinear and asymmetric, and that modeling should include random and dynamic qualities of arcing. Emanuel model is based on laboratory measurements and theoretical components [1] suggested two dc sources connected anti-parallel with two diodes to simulate zero periods of arcing and asymmetry as seen in Figure 3.

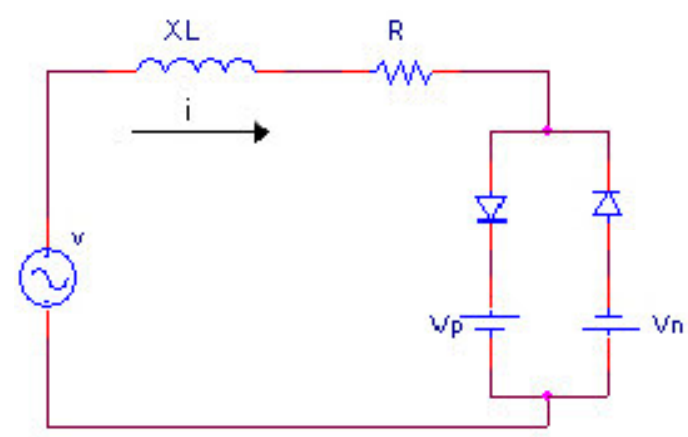

Figure 3. The Emanuel arc model

In [5] for consideration of nonlinearity in earth impedance the arcing high impedance fault was modeled as two sets (positive and negative) of diodes in series with a resistance and a de source Figure 4 illustrates that model.

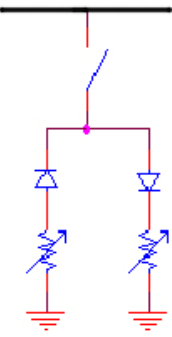

Figure 4. HIF model introduced by [5]

Reference [4] proposed two series time-varying resistances (TVRs) controlled by Transient Analysis of Control Systems (TACS) in EMTP are employed for modeling HIF. One TVR is used for nonlinearity and asymmetry from the voltage-current characteristic for one cycle in the steady state after a fault, then, the other TVR is used to represent buildup and shoulder characteristics from the waveforms in the transient state after HIFas seen in Figure 5

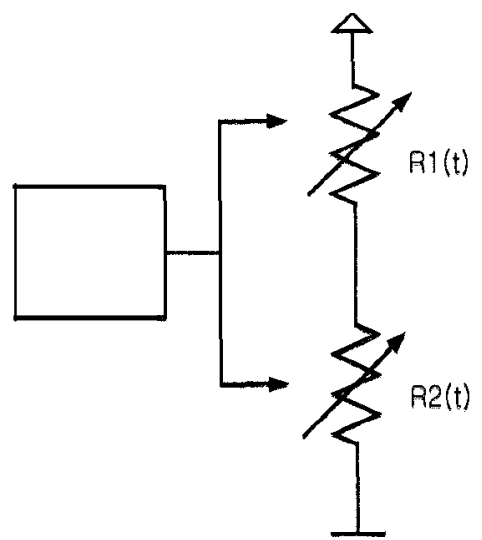

Figure 5. Modeling of HIF as two series connected TVRs

Based on the arc theory, a realistic model of HIF embracing nonlinear impedance, time-varying voltage sources and transient analysis of control system (TACS), controlled switch, as shown in Figure 6, was introduced in [6].

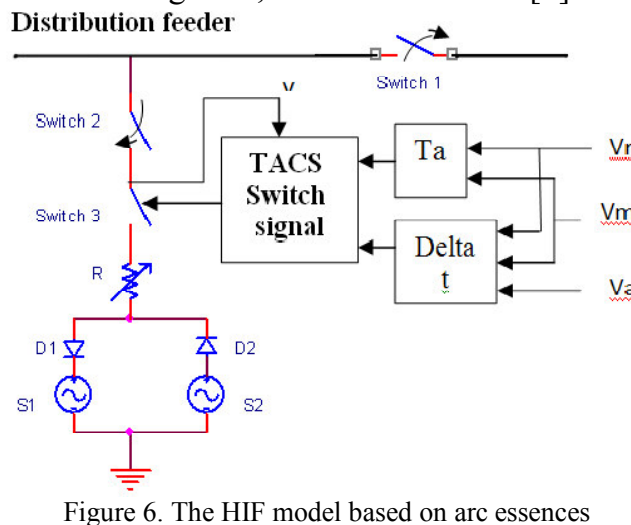

Figure 6. The HIF model based on arc essences

A simplified Emanuel model was introduced in [7]. As shown in Figure 7 the model has two unequal resistances that represent asymmetric fault currents. The two resistances, $R \mathrm{p}$ and $R \mathrm{n}$, represent the fault resistance: unequal values allow for asymmetric fault currents to be simulated. 


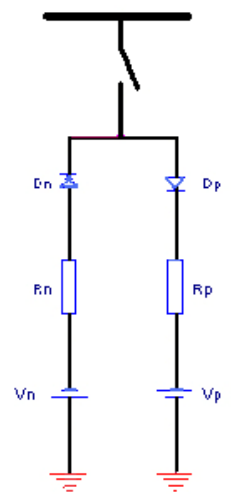

Figure 7. The introduced HIF model in [7]

A simplified 2-diode HIF model was introduced in [8], as shown in Figure 8. This model consists of a nonlinear resistor, two diodes and two de sources that change amplitudes randomly every half cycle.

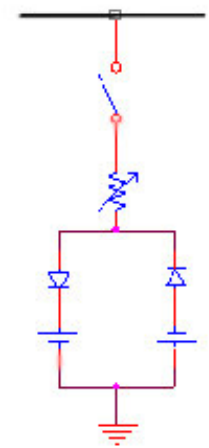

Figure 8 . The HIF model proposed by [8]

HIF model was introduced in [9], as shown in Figure 9. It contains two diodes and polarizing ramp voltages to control arc ignition instants. The arc model consists of linear resistor (representing the ground path resistance), the nonlinear time varying resistor $\mathrm{r}(\mathrm{t})$ (representing the dynamic arc) as well as $\mathrm{DC}$ and $\mathrm{AC}$ sources. The sources ensure asymmetry of the arc current and voltage (DC sources) and variable arc ignition and quenching point (AC sources).

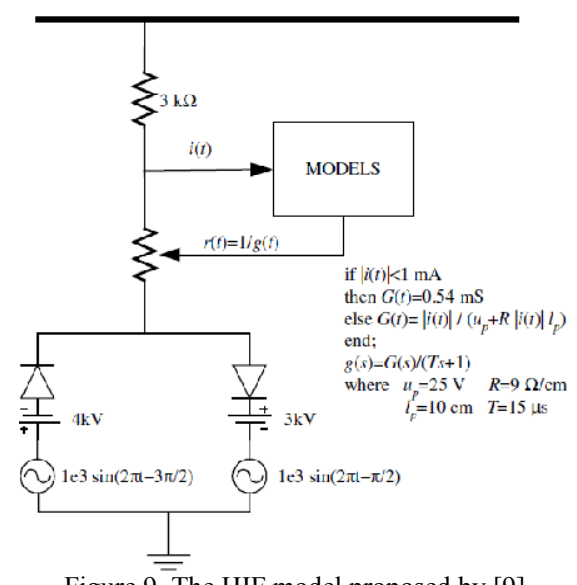

Figure 9. The HIF model proposed by [9]
Based on Emanuel model the authors in [10] proposed a model used several arc models in parallel where their combination produced HIF current. Figure 10 shows this HIF model. Random state of HIF is shown using STATISTIC switch in EMTP. The state of on and off one arc is shown in Figure 10 in the sixth arc model.

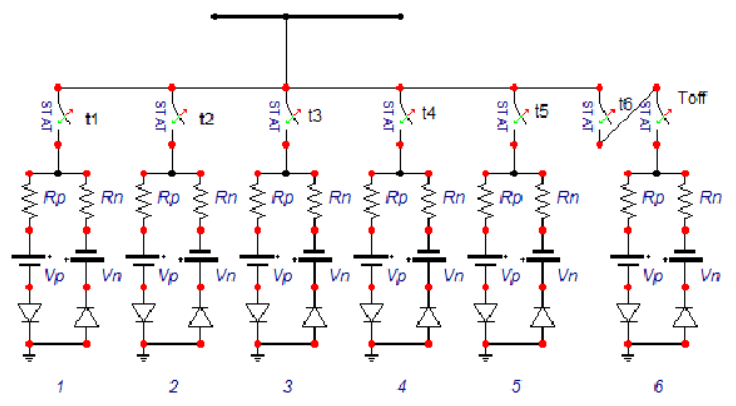

Figure 10. HIF model based on several Emanuel arc model

Many authors have worked on HIF model theory and the dynamics of voltages and currents in HIF electric arc. Most of the studies were based on laboratory studies. As shown in above figures, many models were introduced and developed based on Emanuel arc model [1] and researchers have tried to complete it and come up to a better model for HIF. Also in this paper a new HIF model is introduced.

\section{THE PROPOSED MODEL OF AN ARCING HIF}

The high impedance fault model proposed by this paper shown in Figure 11 includes two DC sources, $V_{p}$ and $V_{n}$, which represent the arcing voltage of air in soil and/or between trees and the distribution line; two resistances, $R_{p}$ and $R_{n}$, between diodes which represent the resistance of trees and/or the earth resistance, and since most observed arcs occur in highly inductive circuits [11] two inductances, $L_{p}$ and $L_{n}$ were added to the circuit. The effect of the inductances leads to the nonlinearity loop shape in the $V-I$ curve and the desired asymmetrical shape for the HIF current. When the line voltage is greater than the positive DC voltage $V_{p}$, the fault current starts flowing towards the ground. The fault current reverses backward from the ground when the line voltage is less than the negative DC voltage $V_{n}$. In the case when the line voltage is in between $V_{p}$ and $V_{n}$, the line voltage is counter-balanced by $V_{p}$ or $V_{n}$ so that no fault current flows. Typical fault current and $V-I$ curves are shown in Figures 12 and 13

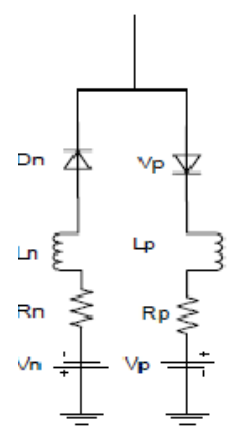

Figure 11. Two diode fault model for a HIF containing $V_{n}, V_{p} R n, R p, L n, L p$ 


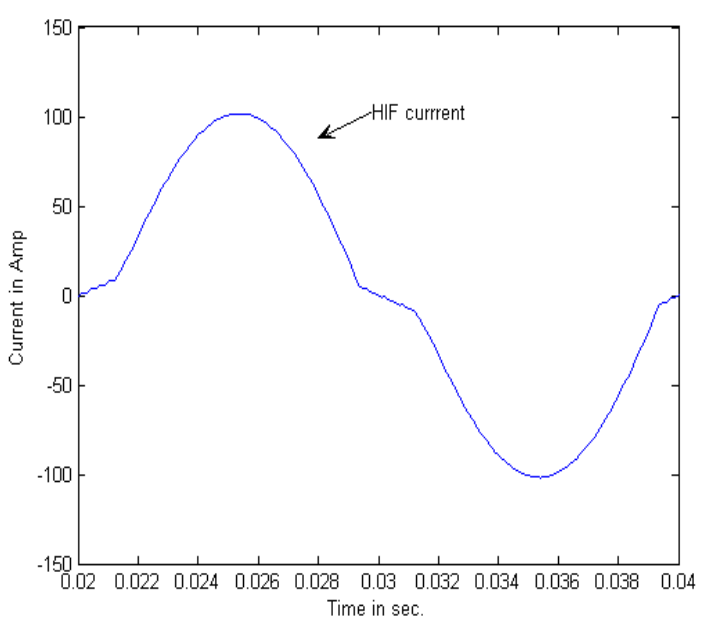

Figure 12. A typical current curve for HIF based on the model of Figure 11

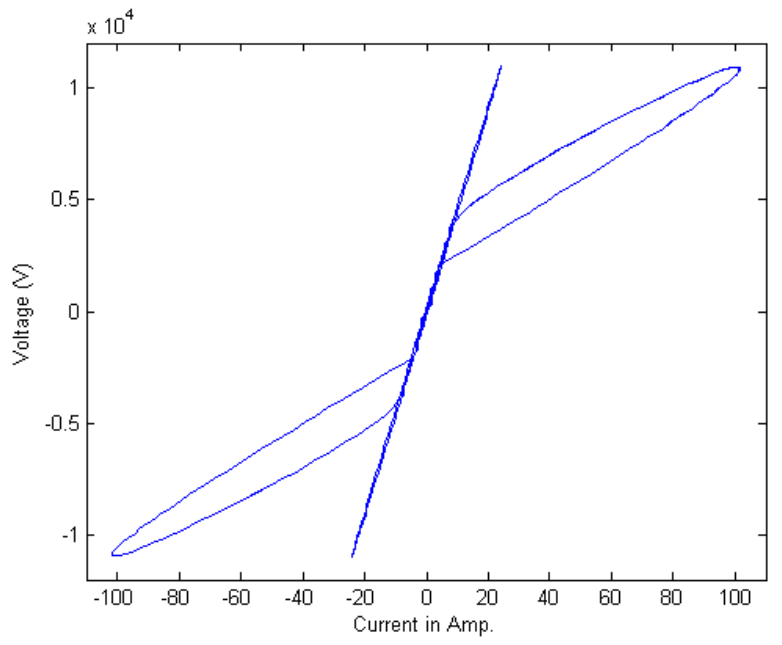

Figure 13. A typical voltage-current characteristic of HIF produced by the proposed model
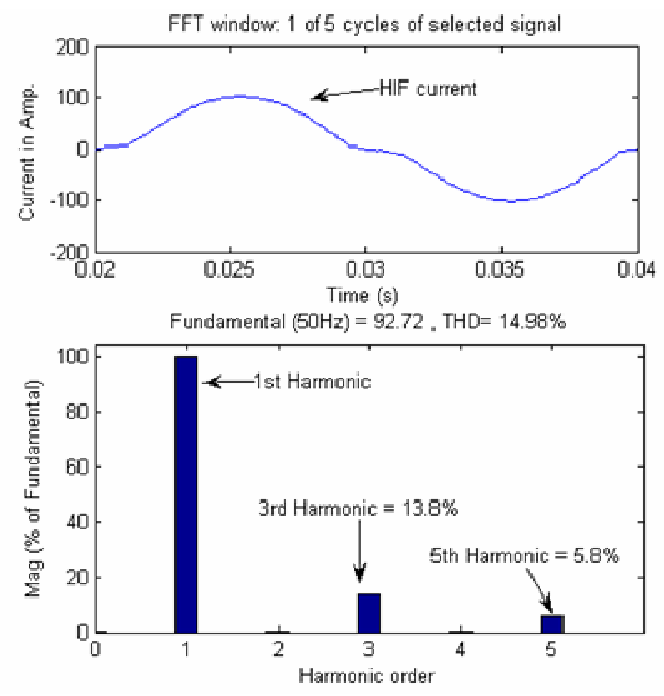

Figure 14. Harmonic content of the HIF model
The HIF current harmonic content produced by the proposed model is analysed and shown in Figure 14. The 3rd and the $5^{\text {th }}$ harmonics are $13.8 \%$ and $5.8 \%$, respectively, with respect to the fundamental current. Comparing the results with the analysed KEPCO data done by [12], the results from the HIF model above shows an encouragingly good match, especially in the harmonic content. In other words, the proposed model is capable of reproducing the experimental HIF results reported by [4] and analysed by [12]. It is also important that the adjustable parameters of the model allow variation of the resultant HIF characteristics.

\section{CONCLUSIONS}

The aim of this paper is to develop a model that represents and predicts all important high impedance fault characteristics, including nonlinearity, asymmetry and harmonic content. The physical processes occurring in an arc result in a unique characteristic signature. Accordingly, a new model for a high impedance fault has been proposed and tested, containing active as well as passive elements (voltage sources, diodes, resistances and inductances), giving a very satisfactory representation of the arc characteristics. The new model preserves the unique shape of the high impedance fault voltage and current, and it also has the harmonics content, consistent with experimentally observed behavior. Thus the proposed model can be considered an appropriate and physically well justified representation of high impedance fault characteristics and can be harnessed to generate various data necessary for developing more reliable HIF detecting algorithms.

\section{REFERENCES}

[1] A. E. Emanuel, D. Cyganski, J. A. Orr, S. Shiller, and E. M. Gulachenski, "High impedance fault arcing on sandy soil in $15 \mathrm{kV}$ distribution feeders: contributions to the evaluation of the low frequency spectrum," IEEE Transactions on Power Delivery, vol. 5, pp. 676-86, 1990

[2] M. B. Djuric and V. V. Terzija, "New approach to the arcing faults detection for fast autoreclosure in transmission systems," IEEE Transactions on Power Delivery, vol. 10, pp. 1793-1798, 1995.

[3] A. T. Johns, R. K. Aggarwal, and Y. H. Song, "Improved techniques for modelling fault arcs on faulted EHV transmission systems," IEE Proceedings Generation, Transmission and Distribution, vol. 141, pp. 148-154, 1994..

[4] S. R. Nam, J. K. Park, Y. C. Kang, and T. H. Kim, "A modeling method of a high impedance fault in a distribution system using two series timevarying resistances in EMTP," presented at Proceedings of Power Engineering Society Summer Meeting, 15-19 July 2001, Vancouver, BC, Canada, 2001.

[5] A.M. Sharaf, L.A. Snider, K. Debnath, "A neural network based back error propagation relay algorithm for distribution system high impedance fault detection" Advances in Power System Control, Operation and Management, 1993. APSCOM-93., 2nd. International Conference on , pp. $613-620,1993$

[6] David Chan Tat Wai; Xia Yibin "A novel technique for high impedance fault identification" Power Delivery, IEEETransactions on, Vol.13 , pp. $738-744$, July 1998.

[7] T.M. Lai, L.A. Snider, E. Lo. "Wavelet Transform Based Relay Algorithm for the Detection of Stochastic High Impedance Faults" International Conference on Power System Transient, in New Orland, pp. 1-6,IPTS 2003 
[8] Y. Sheng, S.M. Rovnyak, "Decision Tree-Based Methodology for High Impedance Fault Detection" Power Delivery, IEEE Transactions on, Vol.19, Issue: 2, pp.533 - 536, April 2004.

[9] M. MICHALIK, W. REBIZANT, M. LUKOWICZ, S.-J. LEE, KANG: "Wavelet Transform Approach to High Impedance Fault Detection in MV Networks", Proceedings of the 2005 IEEE PowerTech Conference, St. Petersburg, Russia, CD-ROM, paper 73, June 2005.

[10] A. R. Sedighi, M. R. Haghifam, "Simulation of High Impedance Ground Fault In Electrical Power Distribution Systems" International Conference on Power System Technology 2010

[11] D. I. Jeerings and J. R. Linders, "Ground resistance-revisited," IEEE Transactions on Power Delivery, vol. 4, pp. 949-56, 1989.

[12] Naser Zamanan "A novel methodology for on-line identification of arcing high impedance faults on overhead power distribution feeders" $\mathrm{PhD}$ dissertation, School of Electronics and Computer Science, Electrical Power Engineering Group, University of Southampton, UK, 2008 